The work was supported by the Russian Government Program for the Recruitment of the leading scientists into the Russian Institutions of Higher Education 14. W03.31.0029.

doi: http://dx.doi.org/10.7124/bc.000A00

\section{T-3. Optimization of in vitro model for analysis of tumor cell migration dynamics}
A. O. Kravchenko ${ }^{1,2}$, V. R. Ko- sach $^{1}$, K. A. Shkarina ${ }^{1}$, I. V. Zaiets ${ }^{1,2}$, I. O. Tykhonkova $^{1}$, A. I. Khoruzhenko ${ }^{1}$
${ }^{1}$ Institute of Molecular Biology and Genetics, Kyiv, Ukraine, 03143; ${ }^{2}$ Educational and Scientific Center "Institute of Biology and Medicine", Taras Shevchenko National University of Kyiv 64/13, Volodymyrska Str., Kyiv, Ukraine, 01601 tykhonkova@gmail.com NAS of Ukraine 150, Akademika Zabolotnoho Str.,

Migration ability is an important feature of tumor cells. There are several approaches to analyze the dynamics of cancer cell migration in vitro. One of the most perspective and closer to the in vivo conditions is the model of initiation of the cell migration from 3D multicellular spheroids onto growth surface. Aim. Optimization of the model for adequate quantitative characteristics of the tumor cell locomotion during several days. Methods. 2D and 3D MCF-7 cell culture, immunofluorescence analysis, and image analysis using computer software Fiji. Results. Unification of spheroid size allowed avoiding a significant data deviation. The obtained spheroids spread completely for 3 days. The highest migration ratio was observed at the 2nd day. The proliferation level at each of 3-day experiment was the same and did not exceed $3 \%$. The validity of the model was tested after migration inhibition by rapamycin (mTOR signaling inhibitor). Additionally, this model was successfully applied to immunofluorescence analysis, namely investigation of p85S6K1 subcellular localization in moving MCF-7 cells. Conclusions. Double filtration of multicellular spheroids allowed unification of their size, which promotes an adequate interpretation of the migration assay. This model enabled the study of tumor cells migration dynamics and can be further used for the development of anticancer drug.

doi: http://dx.doi.org/10.7124/bc.000A01

\section{U-1. The involvement of DNA damage response pathway in nuclear reorganization during netotic initiation}

Burhan Uyanik $^{1}$, Bogdan B. Grigorash ${ }^{1,2}$, B. Uyanik ${ }^{1}$, Oleg N. Demidov ${ }^{1,2}$

${ }^{1}$ INSERM 1231, University of Burgundy FrancheComte, Dijon, France; ${ }^{2}$ Institute of Cytology RAS, Saint-Petersburg, Russia uyanik.burhan@hotmail.fr

The netotic type of cell death plays an important role in innate immunity and host defense. It is characterized by epigenetic changes in chromatin modifications, nuclear envelope disassembly, chromatin expulsion and formation of extracellular chromatin nets loaded with anti-bactericidal granules containing aggressive proteinase and pro-oxidant enzymes. Recently, it was shown by several groups that this process has a negative impact on tumor progression. Methods: To study 